\title{
The impact of intermittency on bed load sediment transport
}

\author{
Santiago J. Benavides ${ }^{1, *}$, Eric A. Deal ${ }^{1,2}$, Matthew Rushlow ${ }^{1}$, Jeremy G. Venditti ${ }^{3}$, Qiong \\ Zhang $^{4}$, Ken Kamrin ${ }^{4}$, and J. Taylor Perron ${ }^{1}$
}

${ }^{1}$ Department of Earth, Atmospheric, and Planetary Sciences, Massachusetts Institute of Technology, Cambridge, MA 02139, USA

${ }^{2}$ Present Address: Department of Earth Sciences, Engineering Geology, ETH Zurich, Zurich, Switzerland

${ }^{3}$ School of Environmental Science and Department of Geography, Simon Fraser University, Burnaby, British

Columbia V5A 1S6, Canada

${ }^{4}$ Department of Mechanical Engineering, Massachusetts Institute of Technology, Cambridge, MA 02139, USA

*e-mail: santib@mit.edu, phone: +1 (512) 825-8931

This EarthArXiv preprint has been submitted for publication in PNAS. It has not been peer-reviewed. Subsequent versions of this manuscript may have slightly different content. If accepted, the final version will be available via the "Peer-reviewed Publication DOI" link on the EarthArXiv webpage. Please feel free to contact the authors with any feedback.

Classification

Physical Sciences: Earth, Atmospheric, and Planetary Sciences

Keywords

sediment transport, intermittency, stochastic, bed load, on-off intermittency, flume experiment

\begin{abstract}
Sediment transport by wind or water near the threshold of grain motion is dominated by rare transport events. This intermittency makes it difficult to calibrate sediment transport laws, or to define an unambiguous threshold for grain entrainment, both of which are crucial for predicting sediment transport rates. Intermittency in sediment transport has been observed in many contexts, but few studies have attempted to explain its origins or its impact on transport rates. Here we present a model that captures this intermittency and show that the noisy statistics of sediment transport contain useful information about the sediment entrainment threshold and the variations in driving fluid stress. Using a combination of laboratory experiments and analytical results from the study of stochastic systems we determine the threshold for grain entrainment in a novel way that is independent of any previous method. Furthermore, our analysis reveals a new property, the "bed sensitivity", which can be used to predict conditions under which transport will be intermittent. Our work suggests strategies for improving measurements and predictions of sediment flux and hints that the sediment transport law may change close to the threshold of motion.
\end{abstract}

\section{Significance}

Sediment transport by wind or water is intermittent - displaying long periods of low transport followed by sudden large transport events. Intermittency is commonly seen as unwanted noise that makes it difficult to predict sediment flux. We uncover why and when intermittency occurs, and we show that the noisy statistics of intermittent sediment transport encode information about the threshold of sediment motion and the variations in driving fluid forces. This knowledge can help improve the accuracy of sediment transport predictions, which are central to many engineering and geological applications. The ingredients necessary for intermittency - noise near a transition - apply to many systems beyond sediment transport.

\section{Introduction}

Sediment transport by wind and water shapes many of Earth's landscapes. Models that predict how rapidly a flow can move sediment are essential for understanding the evolution of Earth's surface ${ }^{1,2}$, mitigating risks posed by natural hazards, designing engineering structures that will interact with moving sediment ${ }^{3-5}$, and restoring landscapes that have been modified by human activities $^{6-8}$. Most sediment transport models consist of empirical formulas used to estimate the time-averaged sediment flux ${ }^{9-18}$. These formulas use fluid and bed properties, such as the time-averaged fluid shear stress at the bed and the critical shear stress necessary for grain motion, to estimate the sediment flux. A crucial component of this relation is the critical shear stress, below which little or no sediment transport occurs ${ }^{15,19}$. Most transport in gravel-bedded rivers occurs near this threshold, 
when fluid stresses are just able to dislodge grains ${ }^{20,21}$, and involves grains rolling and hopping along the bed without becoming suspended in the fluid, a regime known as bed load transport.

Sediment flux near the threshold of motion is intermittent - characterized mostly by low transport, but punctuated by short, rare events in which the instantaneous sediment flux is much larger than the mean. Intermittency poses a challenge for calculating time averages and thus contributes to large uncertainties in predictions of bed load sediment flux, in part because the critical shear stress becomes difficult to measure ${ }^{22}$. Intermittency has been observed in sediment flux driven by wind ${ }^{23,24}$ and water, both laminar ${ }^{25}$ and turbulent ${ }^{26-34}$. Intermittency increases the convergence time - the length of the averaging window necessary for calculating the true value of the mean sediment flux ${ }^{29,35}$. Convergence times of tens of hours have been observed in experiments at low transport rates ${ }^{36}$. This makes it difficult to accurately calculate the average flux as a function of the average shear stress, which is the basis for most estimates of the critical shear stress ${ }^{22}$. More generally, intermittency makes it difficult to define universal sediment transport laws.

Few studies of bed load transport have attempted to account for intermittency. While the observation of noise in sediment flux time series has inspired probabilistic models of sediment transport ${ }^{10,27,28,36-40}$, these studies commonly ignore correlations between fluctuating variables, an approximation that breaks down as the critical shear stress is approached ${ }^{27,36,37,39}$. It is therefore problematic to use such models to investigate intermittency in sediment transport near the threshold of motion.

Here we present a model of bed load sediment transport that reproduces intermittency by explicitly accounting for these correlations, and in doing so provides us with a method of calculating the critical shear stress that utilizes the intermittency rather than avoiding it. We first present a series of laboratory flume experiments and show how the intermittency of sediment flux increases as the threshold of motion is approached. We then propose a dynamical equation for sediment flux that includes a stochastic term that describes the noise in bed shear stress. This equation makes predictions that are consistent with bed load transport statistics in flume experiments. We show how to use our model to extract valuable information from a noisy bed load time series. This includes two new independent ways to estimate the critical shear stress and a method for calculating the distribution of waiting times between sediment transport events, which can be used to estimate the convergence time at other shear stress values. We also predict the range of shear stress at which sediment flux will be strongly intermittent, and introduce a new property called the "bed sensitivity" that quantifies the shear stress fluctuations experienced by a particular bed configuration. In addition to aiding predictions of bed load transport, our model implies that widely used empirical bed load transport formulas should be modified close to the threshold of motion, where coarse sediment transport most commonly occurs in gravel bedded rivers ${ }^{20,21}$.

\section{Intermittent bed load time series}

We performed a series of experiments in a narrow flume under bed load transport conditions (Figure 1a, Methods). In each run we set the sediment feed rate and water discharge, allowed the sediment bed to aggrade until the bed reached a constant slope angle and the sediment flux out of the downstream end of the flume equaled the sediment feed rate, and then captured the motions of grains from the side using a high-speed camera. We used two types of grains: glass spheres $5 \mathrm{~mm}$ in diameter (Figure $1 \mathrm{~b}$ ), and natural river sediment sieved to include intermediate diameters between $4.0 \mathrm{~mm}$ and $5.6 \mathrm{~mm}$ (Figure 1c). Image frames from each experiment were then analyzed with a grain detection and tracking algorithm yielding grain positions, tracks, and velocities for each frame (Figure 1b,c; Methods). For each experiment we measured two quantities, (i) a time series of the instantaneous non-dimensional downstream sediment flux, $q^{*}$, and (ii) the time-averaged non-dimensional shear stress, $\left\langle\tau^{*}\right\rangle$, also sometimes referred to as the Shields Number, where $\langle\cdot\rangle$ denotes a time average (Methods). The non-dimensional shear stress gives an estimate for the ratio of fluid drag and lift forces ${ }^{19}$, which act to move grains, to the submerged weight of grains, which acts to resist motion.

Figures 2a-2c show snapshots of three different glass sphere runs with low, intermediate, and high time-averaged sediment flux, corresponding to progressively increasing time-averaged shear stress. A sample of the sediment flux time series for each of these runs is shown in Figures 2d-2f. Each time series features periods of low transport followed by bursts in transport, the tell-tale sign of intermittency. This is more pronounced for the low shear stress values, consistent with previous observations that intermittency increases as the shear stress decreases towards the threshold of motion ${ }^{26-30,33,34,40}$. The same behavior is seen for the natural grain experiments (Extended Data Figure 1).

We quantify the intermittency by calculating probability density functions (PDFs) of the sediment flux (Figure 2g). Whereas a non-intermittent time series of a fluctuating signal would typically display a Gaussian PDF (grey dashed line, Figure 2g), an intermittent time series spends most of its time at values much smaller than the average, with occasional bursts of transport at rates larger than the average, and hence its PDF is skewed to small values. The more intermittent the time series is, the more time it spends close to zero, and the larger the values of the PDF would be at small values of $q^{*}$. Our flume experiments show exactly this trend: experiments with lower average shear stress have more intermittent time series of $q^{*}$ (Figure 2d-f) and PDFs of $q^{*}$ with steeper negative slopes at small values of $q^{*}$ (Figure $2 \mathrm{~g}$ ). In the next section we describe a model of bed load transport that captures these properties of intermittent sediment flux and enables better characterization and prediction of 

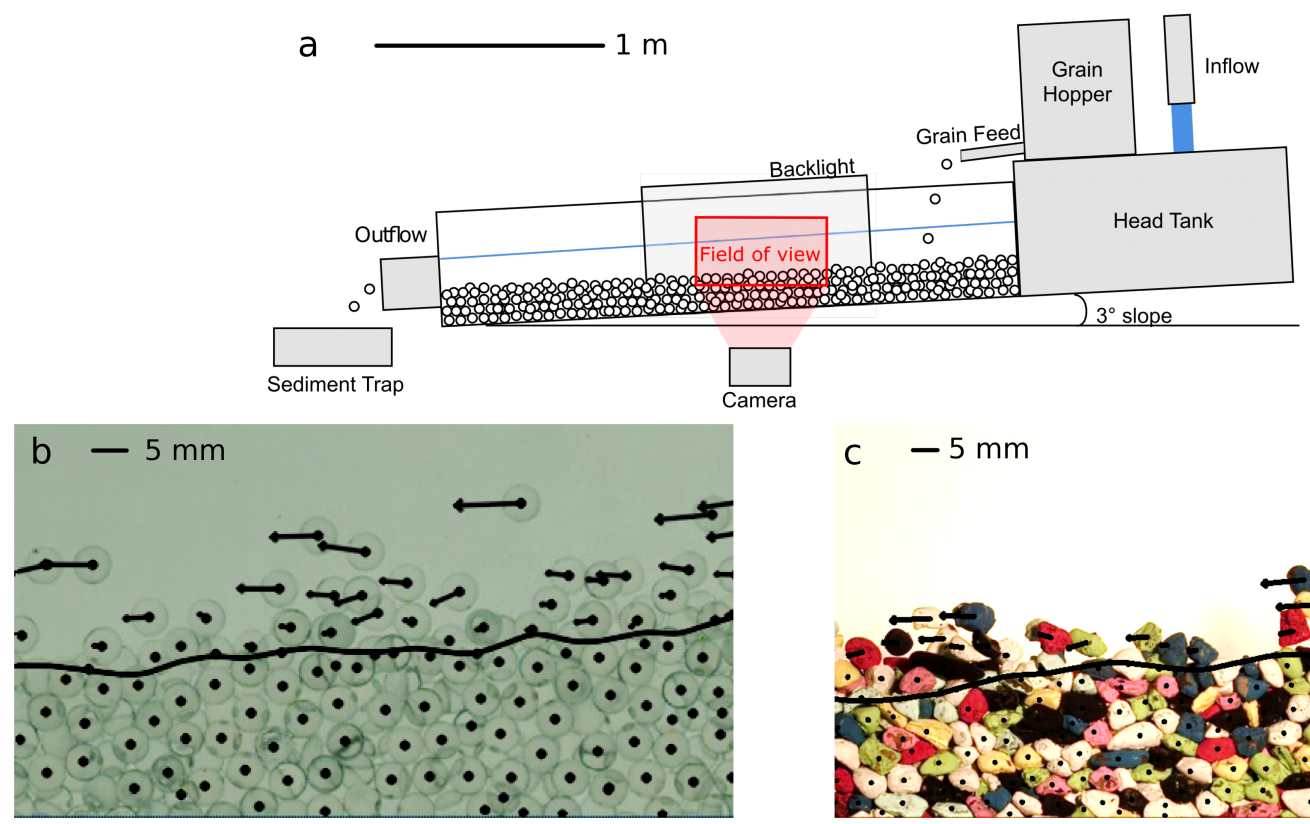

C $\quad-5 \mathrm{~mm}$

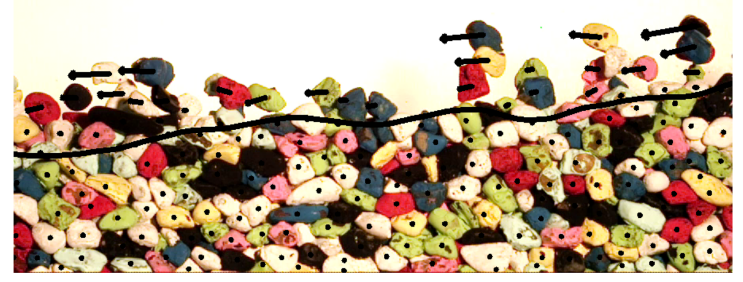

Figure 1. Experimental setup and grain tracking. a, Schematic side view of laboratory flume. b Image frame from an experiment using glass spheres, with particle centers and velocities calculated from grain tracking overlain (Methods). Arrow lengths indicate velocity magnitude. The thick solid line indicates the top of the bed, below which grains were stationary over a sufficiently long time (Methods). c, Same as b, but for an experiment using natural grains. Grains have been painted to aid grain tracking.

sediment transport near the threshold of motion.

\section{Stochastic model of sediment transport near the threshold of grain motion}

Intermittent time series displaying bursting behavior are seen in many systems undergoing a transition from an inactive to an active state. This particular kind of intermittency is called on-off intermittency ${ }^{41-43}$ and has been observed in systems ranging from chemical reactions to turbulent magnetic field amplification ${ }^{4-54}$. The key ingredient for on-off intermittency is multiplicative noise (a noisy growth rate) at the onset of a bifurcation, so that the solution experiences both exponential decay and growth. Following previously established results from the study of such systems, we developed a model for the dynamics of sediment transport near the threshold of motion.

We expect the dynamical equation for $q^{*}$ to have the form $d q^{*} / d t^{*}=\mathscr{N}\left(q^{*}\right)$, where $t^{*}$ is a dimensionless time (Methods) and $\mathscr{N}\left(q^{*}\right)$ is a nonlinear operator that depends implicitly on the time-averaged shear stress $\left\langle\tau^{*}\right\rangle$, the critical shear stress $\left\langle\tau^{*}\right\rangle_{c}$, and the temporal statistics of the shear stress. Although $\mathscr{N}\left(q^{*}\right)$ is not known, we approximated it using a standard approach in the study of bifurcations and phase transitions ${ }^{55,56}$. This procedure amounts to assuming that the sediment flux close to the threshold of motion is very small $\left(q^{*} \ll 1\right)$, allowing one to approximate the dynamical equation with only a few polynomial terms which are dominant in that regime (Methods). By further requiring that nonzero sediment transport occurs for $\left\langle\tau^{*}\right\rangle>\left\langle\tau^{*}\right\rangle_{c}$, the resulting expression for the nonlinear operator is $\mathscr{N}\left(q^{*}\right) \approx\left(\left\langle\tau^{*}\right\rangle-\left\langle\tau^{*}\right\rangle_{c}\right) q^{*}-\beta\left(q^{*}\right)^{2}$, where $\beta$ is a positive dimensionless constant coefficient that can be determined empirically with measurements, but which does not enter into our results (Methods). We then include a stochastic, zero-mean noise term $\xi$, that represents fluctuations in the shear stress (which can arise from fluctuations of turbulent fluid motion or irregular bed protrusions that can enhance local shear stress). The final model equation is then

$$
\frac{d q^{*}}{d t^{*}}=\left(\left\langle\tau^{*}\right\rangle-\left\langle\tau^{*}\right\rangle_{c}+\xi\right) q^{*}-\beta\left(q^{*}\right)^{2} .
$$

To understand how Equation (1) produces intermittent sediment flux, note that, for small $q^{*}$, it admits an exponential solution with a growth rate equal to the noisy shear stress offset, $\left\langle\tau^{*}\right\rangle-\left\langle\tau^{*}\right\rangle_{c}+\xi$. If the shear stress is larger than the critical value, the time-averaged flux $\left\langle q^{*}\right\rangle$ will be positive. Despite this, at times when the noise $\xi$ is sufficiently negative such that $\left\langle\tau^{*}\right\rangle-\left\langle\tau^{*}\right\rangle_{c}+\xi$ is negative, the sediment flux will decay exponentially towards zero. As the noise changes and $\left\langle\tau^{*}\right\rangle-\left\langle\tau^{*}\right\rangle_{c}+\xi$ 

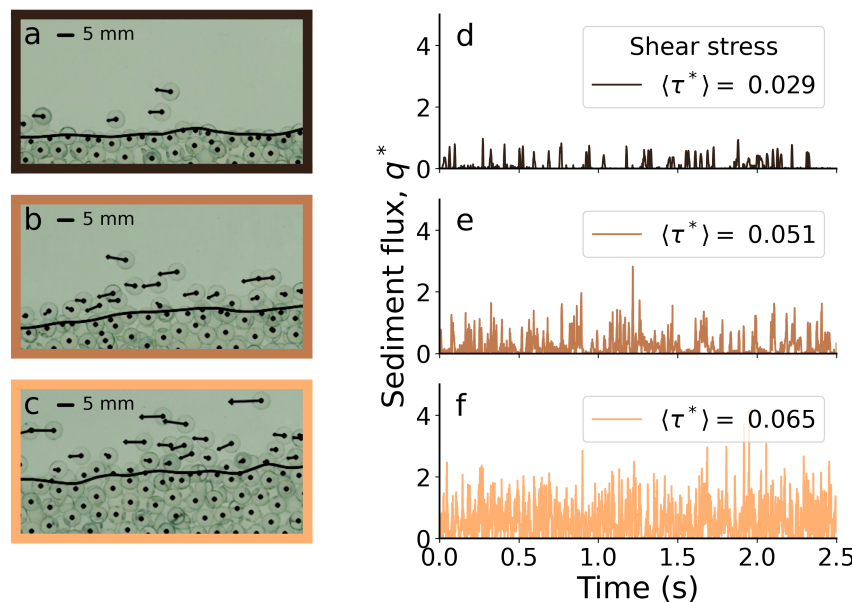

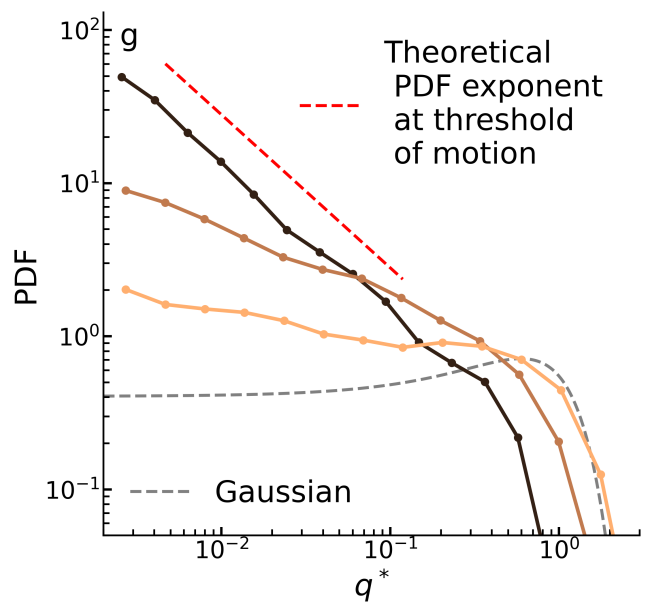

Figure 2. Intermittent bed load sediment flux. Sample data from three flume experiments using glass spheres, representing typical runs with low sediment flux (a and $\mathbf{d}$ ), intermediate sediment flux (b and $\mathbf{e}$ ), and high sediment flux (c and f). a-c, High-speed video frames showing grain centers and velocities and the location of the bed, as in Figure 1b. d-f, Samples of the corresponding sediment flux time series. g, Probability density function (PDF) calculated from the sediment flux time-series. The red dashed line shows the theoretical exponent of the PDF tail, -1 , at the threshold of motion. The grey dashed line shows a Gaussian distribution with the same mean and variance as the high sediment flux case.

becomes positive again, the sediment flux will grow towards (and sometimes beyond) $\left\langle q^{*}\right\rangle$. This repeated growth and decay of the sediment flux is the intermittency. Equation (1) also produces less intermittency for larger $\left\langle\tau^{*}\right\rangle-\left\langle\tau^{*}\right\rangle_{c}$ (as illustrated in Figure 2d-f), because this makes it more rare for the noise to be large enough to cause the sediment flux to decay.

Stochastic analysis of equation (1) (Methods) makes two useful predictions about bed load transport near the threshold of grain motion that we can test with our experimental data. First it predicts the shape of the PDF of $q^{*}$ at steady state. Although the functional form of the full PDF is known, we consider only its "tail" at small values of $q^{*}$, which is predicted to be a power-law with an exponent that depends on the shear stress offset:

$$
\operatorname{PDF}\left(q^{*}\right) \propto\left(q^{*}\right)^{\left(\left\langle\tau^{*}\right\rangle-\left\langle\tau^{*}\right\rangle_{c}\right) / S-1},
$$

where $S=\int_{0}^{\infty} \overline{\xi(0) \xi\left(t^{* \prime}\right)} d t^{* \prime}$ is the autocorrelation of the noise, with the overbar denoting an average over different realizations of the noise. Equation (2) predicts that the exponent of the power-law tail of the PDF becomes more negative as the critical shear stress is approached, reaching a minimum value of -1 when $\left\langle\tau^{*}\right\rangle=\left\langle\tau^{*}\right\rangle_{c}$. This implies larger and larger probabilities for small values of sediment flux, consistent with the observation that sediment flux in our experiments becomes more intermittent as the critical shear stress is approached (Figure 2d-f). This prediction is also consistent with the PDFs of $q^{*}$ from our experiments (Figure 2g), which have power-law tails with exponents that become more negative as average shear stress decreases and the intermittency increases. The most intermittent case has a PDF tail exponent of approximately -1 , the theoretical value at the threshold of motion.

Second, our stochastic model predicts how rare the intermittent bursts of sediment flux are. As the shear stress approaches the threshold of motion, long waiting times between transport events of a certain size become more likely and the bursts of transport occur as more irregular and extreme events. The distribution of waiting times, $\Delta t$, between transport events of an arbitrary threshold $q_{0}^{*}$ (Methods) is ${ }^{42,57}$ :

$$
\operatorname{PDF}(\Delta t) \propto \Delta t^{-3 / 2} e^{-\Delta t / \delta}
$$

provided $q_{0}^{*} \ll 1$, where $\delta \propto S /\left(\left\langle\tau^{*}\right\rangle-\left\langle\tau^{*}\right\rangle_{c}\right)^{2}$ is a characteristic time-scale for the longest waiting times under the specified flow conditions. The proportionality in equation (3) allows us to work with the dimensional waiting time $\Delta t$, instead of its dimensionless counterpart $\Delta t^{*}$, since they are related by a constant (Methods). Hereafter we use the dimensional time $t$ for clarity.

The PDF of waiting time has a power-law tail, but, unlike the PDF of $q^{*}$, the waiting time PDF has an exponent that is always $-3 / 2$, independent of shear stress. Instead, as the shear stress approaches the threshold of motion, long waiting times 
between transport events of a certain size become more likely and the bursts of transport occur as more irregular and extreme events. Figure 3 shows waiting time PDFs for our flume experiments with glass spheres and natural grains, along with the power-law PDF predicted by our stochastic model (Equation (3)) at the threshold of motion. This comparison confirms that,

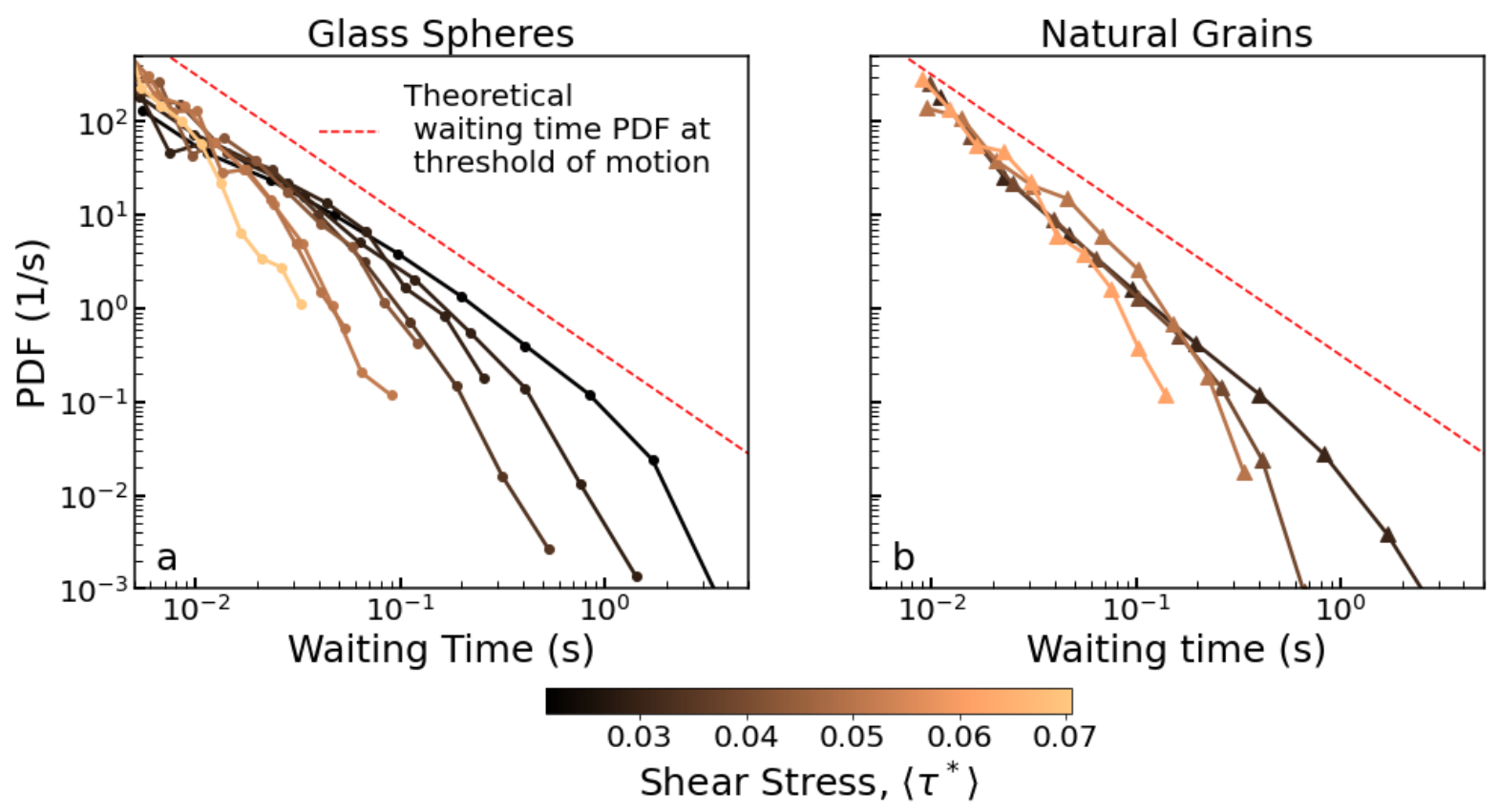

Figure 3. Waiting times between sediment transport events. Probability density functions (PDFs) for waiting times between transport events of size $q_{0}^{*}=0.05$, for various values of shear stress $\left\langle\tau^{*}\right\rangle$, in the experiments with a glass spheres, and b natural grains. The red-dashed line shows the theoretical shape of the waiting time PDF at the threshold of motion.

as the shear stress approaches the entrainment threshold, long waiting times become more likely. Furthermore, experiments with the lowest shear stresses (darkest curves) have PDF tail exponents close to $-3 / 2$. Power-law waiting time distributions consistent with an exponent of $-3 / 2$ have also been observed in other bed load sediment transport experiments using glass spheres $^{28}$ and natural grains ${ }^{34}$ as well as in experiments with wind-blown sand ${ }^{24}$. The ability of our stochastic model to predict probability distributions of both sediment flux and waiting times between transport events suggests that it robustly captures the statistics of intermittent bed load transport near the threshold of motion.

\section{Critical shear stress, bed sensitivity, and average waiting times}

The stochastic model of bed load sediment transport offers new ways to estimate the critical shear stress, and it also reveals a measure of flow-sediment interaction that has not previously been described. Equation (2) predicts that the exponent of the tail of $\operatorname{PDF}\left(q^{*}\right)$ is equal to $\left(\left\langle\tau^{*}\right\rangle-\left\langle\tau^{*}\right\rangle_{c}\right) / S-1$, making it linear in $\left\langle\tau^{*}\right\rangle$ with a slope of $1 / S$ and taking the value of -1 at $\left\langle\tau^{*}\right\rangle_{c}$. Thus, the statistics of intermittent bed load transport carry information about two properties of the system: the critical shear stress $\left\langle\tau^{*}\right\rangle_{c}$ and the quantity $S$. Since the noise term $\xi$ represents temporal fluctuations in the shear stress (Equation (1)), the noise autocorrelation $S$ measures the low frequency variability of shear stress, and in a more informal sense the 'strength' of the noise. We call $S$ the 'bed sensitivity' because variability in bed shear stress can be a result of both flow properties (turbulent fluctuations) and bed properties (bed heterogeneity and grain protrusions). We expect a uniform and flat bed in a less turbulent flow to have low bed sensitivity because the bed feels similar shear stresses throughout, whereas a rough bed in a more turbulent flow might experience larger fluctuations in shear stress and therefore have high bed sensitivity. This analysis assumes that $S$ is not a function of $\left\langle\tau^{*}\right\rangle$, which is consistent with the observation that our experiments can be characterized by a single value of $S$. However, this could break down if bed conditions vary substantially with $\left\langle\tau^{*}\right\rangle$, for example with the growth of dunes or ripples in sand bed rivers or the development of bed structures in gravel bed rivers (clusters, lines and cells) that inhibit entrainment ${ }^{58,59}$.

By performing a series of experiments for different values of $\left\langle\tau^{*}\right\rangle$ and measuring the exponent of the PDF of $q^{*}$ for each experiment, we use the linear relation above to calculate both the critical shear stress $\left\langle\tau^{*}\right\rangle_{c}$ and the bed sensitivity $S$. Experiments with glass spheres and natural grains each define a linear trend (Figure 4), consistent with the prediction of the stochastic model. By fitting this line we calculate $\left\langle\tau^{*}\right\rangle_{c}=0.026 \pm 0.002$ and $S=0.048 \pm 0.003$ for the glass spheres, and $\left\langle\tau^{*}\right\rangle_{c}=0.040 \pm 0.008$ and $S=0.06 \pm 0.01$ for the natural gravel (uncertainties are one standard error of the estimated value). 

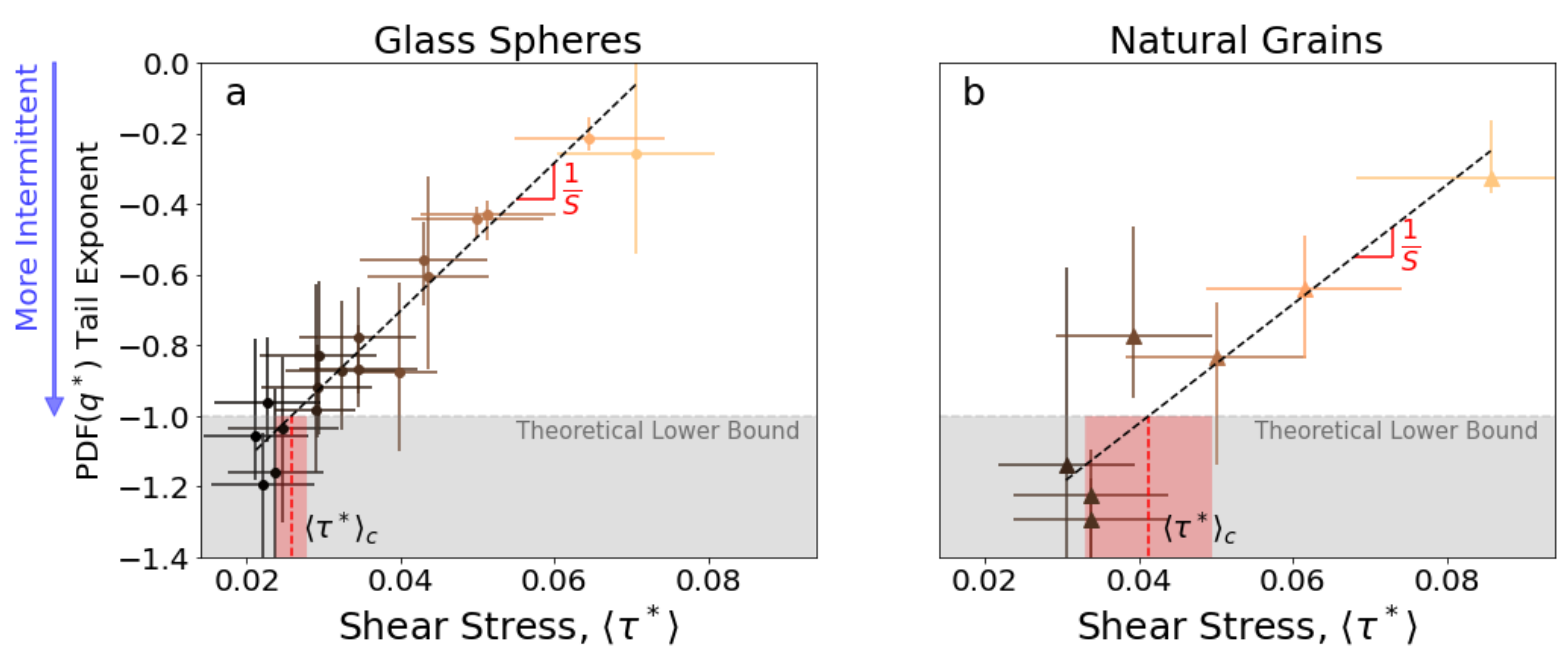

Figure 4. Determination of critical shear stress and bed sensitivity. The tail exponent of $\operatorname{PDF}\left(q^{*}\right)$ for each experiment plotted versus the shear stress. The more negative the exponent, the more intermittent the time series. On-off intermittency predictions state that a minimum exponent of -1 ("theoretical lower bound") occurs at the threshold of motion. a shows data from experiments using glass spheres and $\mathbf{b}$ shows data from experiments using natural grains. Point colors correspond to the shear stress color scale in Figure 3. Vertical uncertainty bars represent minimum and maximum values for exponents measured over a series of smaller intervals of $q^{*}$. The horizontal error bars represent one standard error of the estimated mean shear stress (Methods). Dashed black lines are fits to the data determined by orthogonal distance regression (Methods). Dashed red lines indicate the estimated value of the critical shear stress, which corresponds to the value of the fit at a $\operatorname{PDF}\left(q^{*}\right)$ tail exponent of -1 , rose shading indicating one standard error.

Unlike most previous estimates of $\left\langle\tau^{*}\right\rangle_{c}$, our estimates do not depend on any assumed form of the sediment transport law, yet they are consistent with typical values reported for gravel-bedded rivers ${ }^{22,60,61}$. These are also the first measurements of a new property, the bed sensitivity, $S$. On-off intermittency occurs if the power-law exponent in equation (2) is negative, which requires that $\left\langle\tau^{*}\right\rangle-\left\langle\tau^{*}\right\rangle_{c}<S$. The bed sensitivity therefore determines how close to the threshold of motion a bed load system must be to experience substantial intermittency. If the values of $\left\langle\tau^{*}\right\rangle_{c}$ and $S$ are known, they can be used to predict whether intermittency will be present for a given $\left\langle\tau^{*}\right\rangle$. For example, our results for the glass spheres tell us that intermittency is expected to be present for $\left\langle\tau^{*}\right\rangle<0.074$.

A practical consequence of intermittency is that it requires long time-averages for convergence ${ }^{29,35,36}$, yet it is difficult to know a priori whether or not the averaging time is long enough. Since intermittent bursts of sediment flux dominate the averaging procedure, the convergence time is expected to be proportional to the average waiting time between sediment transport events. From equation (3), the expected waiting time at a specific value of $\left\langle\tau^{*}\right\rangle$ is proportional to:

$$
\langle\Delta t\rangle \propto \frac{\sqrt{S}}{\left\langle\tau^{*}\right\rangle-\left\langle\tau^{*}\right\rangle_{c}} .
$$

The average waiting time between transport events of a certain size thus diverges at the critical shear stress: the closer to the threshold of motion, the rarer transport events become. This would suggest a similar divergence of convergence time, since bursts become rarer. Divergence of the time necessary for a converged average has been observed in laminar ${ }^{25}$ and turbulent ${ }^{33}$ flume experiments, but the functional form of this relation has not previously been predicted or confirmed with experiments. Comparison of the average waiting times between transport events of size $q_{0}^{*}=0.05$ for each experiment with equation (4) (Figure 5) reveals not only that the average waiting time varies as $1 /\left(\left\langle\tau^{*}\right\rangle-\left\langle\tau^{*}\right\rangle_{c}\right)$, as predicted by the stochastic model, but also an agreement between the values of $\left\langle\tau^{*}\right\rangle_{c}$ calculated using the two completely independent methods - the PDF of $q^{*}$ (Figure 4) and the average waiting time (Figure 5) - particularly in the case of glass spheres. This approach could prove useful for both laboratory experiments and field measurements, since it does not require particularly precise measurements of the sediment flux. Indeed, despite state-of-the-art methods, tracking of the natural grains was more difficult and resulted in larger noise levels, particularly in the tail of $\operatorname{PDF}\left(q^{*}\right)$. This caused an apparent disagreement between the two estimates of the critical shear stress for natural grains (Figure $5 \mathbf{b}$ ). We believe that with longer time series, these two estimates would converge, as is seen in the case of the glass spheres. 

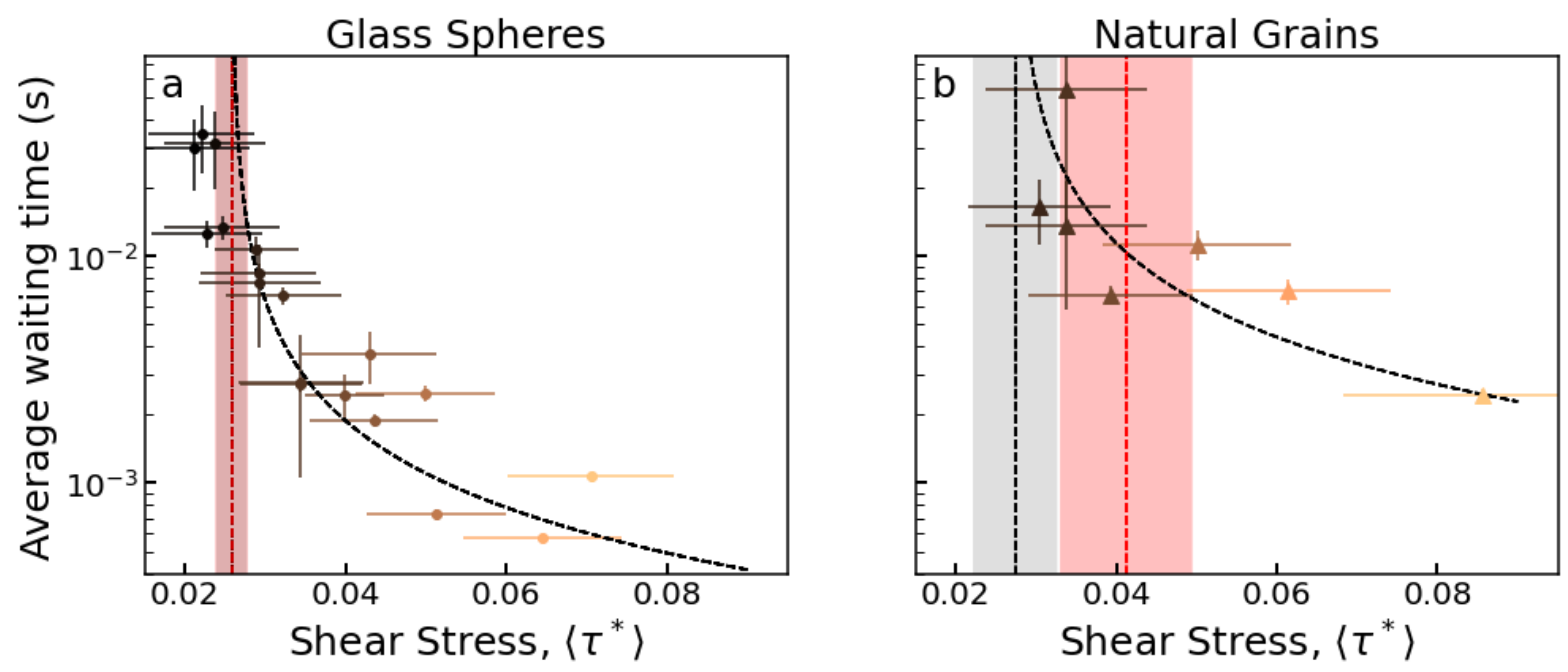

Figure 5. Average waiting times between sediment transport events. The average waiting time between transport events of size $q_{0}^{*}=0.05$ for various values of shear stress $\left\langle\tau^{*}\right\rangle$ in the experiments with a glass spheres, and $\mathbf{b}$ natural grains. Point colors correspond to the shear stress color scale in Figure 3. Uncertainty bars are one standard error of the mean. Dashed black curves are fits of equation (4) to the data using an orthogonal distance regression. Values of the critical shear stress $\left\langle\tau^{*}\right\rangle_{c}$, determined from the fits are denoted by vertical black dashed lines with grey shading indicating one standard error. Red dashed lines with rose shading indicate the critical shear stress determined from the PDF of $q^{*}$ (Figure 4). The black dashed line in panel $\mathbf{a}$ is not visible because the two estimates of the critical shear stress overlap almost exactly.

Convergence times required for a reliable average flux measurement in our experiments ranged from around one second to a few minutes (Extended Data Figure 2). These convergence times are two to three orders of magnitude longer than the average waiting time between transport events (fractions of a second), but both follow the same functional form given by equation (4) (Figures 5 and Extended Data Figure 2). Intermittent sediment flux time series at lower transport stages than our experiments can have even longer convergence times while still displaying similar statistical properties. In Extended Data Figure 3, for example, we show that the experiments of Ancey et al. ${ }^{36}$, which have convergence times of a few hours, are consistent with our intermittent transport model. Given a set of bed load flux time-series and shear stress measurements, the new theory presented here offers a way to find both the critical shear stress and also an estimate for the time-averaging window required for a properly converged average sediment flux. Measuring the convergence time for each experiment and fitting these values to equation (4) would result in an estimate of $\left\langle\tau^{*}\right\rangle_{c}$, and would also provide estimates of convergence times given the extrapolation to smaller (or larger) average shear stress values.

Our results show that a stochastic model of bed load sediment transport close to the threshold of motion reproduces intermittent sediment flux time-series like the ones observed in our experiments as well as in previous studies. Comparing this model with finely resolved sediment flux measurements from grain tracking reveals that there is information in the noise of sediment transport, including two independent ways to estimate the critical shear stress for grain entrainment without fitting a transport law; a new quantity called the bed sensitivity, which determines whether a bed load system will experience intermittency or not; and a way of using waiting times between intermittent transport events to calculate the minimum time required to characterize the average sediment flux. The presence of on-off intermittency should also result in a modified ("anomalous") transport law ${ }^{62,63}$ close to the threshold of motion: the average sediment flux should depend linearly on the shear stress offset, $\left\langle q^{*}\right\rangle \propto\left(\left\langle\tau^{*}\right\rangle-\left\langle\tau^{*}\right\rangle_{c}\right)$, instead of the classical transport law, $\left\langle q^{*}\right\rangle \propto\left(\sqrt{\left\langle\tau^{*}\right\rangle}-\sqrt{\left\langle\tau^{*}\right\rangle_{c}}\right)\left(\left\langle\tau^{*}\right\rangle-\left\langle\tau^{*}\right\rangle_{c}\right)$. This change in transport law could make it difficult to determine the critical shear stress from extrapolation of the classical transport law, a common technique ${ }^{22}$. It could also result in an apparent under-estimation of sediment flux for shear stresses near the threshold of motion, which are typical conditions in gravel-bedded rivers ${ }^{20,21}$.

\section{Materials and Methods}

\section{Laboratory Flume Experiments}

The experiments were performed in the narrow flume facility in the River Dynamics Laboratory at Simon Fraser University in Burnaby, Canada. The experimental setup (Figure 1a) consisted of a flume $2.5 \mathrm{~m}$ long, $45 \mathrm{~cm}$ tall, $1 \mathrm{~cm}$ wide (slightly larger than two grain diameters), which was tilted 3 degrees from horizontal. Water was recirculated at a fixed discharge 
with a pump, with a bulk mean velocity of $u \approx 1 \mathrm{~m} / \mathrm{s}$. The mean water depth was $H=0.1 \mathrm{~m}$ and the mean hydraulic radius was $R=W H /(W+2 H)=0.005 \mathrm{~m}$. This corresponds to a Reynolds number $R e=u R / v \approx 4800$ and a Froude number $F r=u / \sqrt{g H} \approx 1$. Grains were fed into the flume at a fixed rate by a 'tinker feeder' grain hopper ${ }^{64,65}$, making the sediment flux a fixed input parameter in our experiments. Once inside the flume, the grains accumulated, forming an aggrading bed until steady-state was reached, at which point the bed stopped aggrading and grains exited the flume, where they were collected by a sediment trap. Once at steady state, the experimental observations commenced. Data collection consisted of high-speed recordings of the grains from the side (Fig. $1 \mathbf{b}$ and 1c). We focused a series of high-speed Edgertronic cameras on a back-lit region seen in the middle of the schematic in Fig. 1a. This region had approximate dimensions of $14 \mathrm{~cm}$ long by $4 \mathrm{~cm}$ tall and was chosen to be far enough away from both ends of the flume so as to avoid effects from the entrance and exit. Multiple videos were recorded for each experiment, with frame rates varying between different recordings, but were either 520 or 1040 frames per second. Other measurements included: sediment flux by collecting and weighing sediment exiting the flume, steady-state bed slope and water discharge.

For each experiment, instead of specifying the fluid shear stress at the bed, we specified the grain input rate (and thus the average sediment flux). The time-averaged shear stress was then measured once steady state was reached using the 1D momentum balance for shallow-water flow, $\langle\tau\rangle=\rho g R \sigma$, where $\rho$ is the density of water, $g$ the acceleration due to gravity, $R$ the hydraulic radius, and $\sigma$ is the slope of the water taken at steady-state. The slope $\sigma$ was calculated using a linear fit of the measured steady-state water surface. The non-dimensional time-averaged shear stress, i.e. Shields number, was calculated as $\left\langle\tau^{*}\right\rangle=\langle\tau\rangle /\left(\left(\rho_{s}-\rho_{w}\right) D g\right)$.

We performed experiments with two types of grains: uniformly-sized spherical grains (glass beads) with a diameter of $5 \mathrm{~mm}$ and a density of $2550 \mathrm{~kg} / \mathrm{m}^{3}$, and river gravel sieved to yield intermediate diameters between $4.0 \mathrm{~mm}$ and $5.6 \mathrm{~mm}$. We collected data for 18 glass sphere experiments, with $\left\langle q^{*}\right\rangle$ ranging from 0.00395 to 0.876 and $\left\langle\tau^{*}\right\rangle$ ranging from 0.0293 to 0.0766 (Extended Data Figure 4a). We also performed 7 experiments with natural grains, with $\left\langle q^{*}\right\rangle$ ranging from 0.0108 to 0.546 and $\left\langle\tau^{*}\right\rangle$ ranging from 0.0380 to 0.0750 (Extended Data Figure $4 \mathbf{b}$ ).

\section{Grain tracking}

Over the course of the experiments, more than a million image frames were recorded. This large data set required the use of automatic grain tracking. The images were processed using the open-source package OpenCV ${ }^{66,67}$. For the glass spheres, we used an efficient ring finding algorithm to identify individual grains ${ }^{68}$. The algorithm was able to locate $>95 \%$ of all grains in our images. Once the grains had been located by the ring finding algorithm, we tracked them from frame to frame using the python libraries TrackPy ${ }^{69}$ and PIMS ${ }^{70}$. The high frame rate allowed the grains to be accurately tracked. In order to track the natural grains, we painted the grains 8 different colors in equal numbers. The colors were chosen to occupy the 8 corners of a cube in RGB space. We then used a pretrained machine learning algorithm to locate the grains in each image (YOLO2 $\left.{ }^{71}\right)$. The algorithm was primed on a set of 50-100 image-subsets, where each subset contained about 50 grains that had been annotated by hand. The machine learning algorithm found about $75 \%$ of the grains in each image. Once the grains had been recognized in each image, we again used TrackPy and PIMS to link grains between frames. We only linked grains of the same color from frame to frame. The color information reduced the number of possible matches for each grain from one image frame to the next. Both versions of our workflow processed a single frame in less than a second on a normal workstation.

Apart from grain tracking, a 'bed line' was defined at each time. Below this line is the bed, which comprises grains that did not move significantly over the course of $s$ seconds. The bed line at a time $t$ was calculated by averaging image frames from $t-s / 2$ to $t+s / 2$. Any grains that moved significantly in this time interval are effectively averaged away. Once the average was performed, a watershed algorithm was used to automatically find the bed surface and thus define the bed line. For the glass spheres, $s=1.5$ seconds and for the natural grains $s=2$ seconds. Although a definition of a bed was not necessary for any of the theory or calculations done in this work, filtering out grains in the bed (locations below the bed line) proved to significantly reduce noise in the data analysis, discussed in the next section.

\section{Data analysis}

The grain tracking algorithm resulted in positions, tracks, and velocities for every identified grain in each frame. Additionally, the natural grains were given an 'effective' spherical shape with a radius to match their measured density. In this study, we measured time-series of the downstream sediment volume flux per unit flow width, $q_{s}$. To measure this based on our grain tracking data, we did the following for each video: first, we took the average velocity of all grains and rotated the frame so that the average velocity in the vertical direction was zero, leaving only an average velocity in the downstream direction. This accounted for the slope of the channel. Second, we picked a downstream ( $x$-direction) location halfway along the frame for measuring the flux. Any grain intersecting a vertical line at that location was considered in the calculation of the sediment flux. However, we excluded any grains in the bed: those whose centers were more than a grain radius below the bed line. Suppose that, at a time $t$, each grain above the bed that was intersecting the vertical line was enumerated with the index $i$. The sediment flux was then finally computed $q_{s}(t)=\sum_{i} u_{i} A_{i} / b$, where $u_{i}$ is the downstream velocity of the grain, $A_{i}$ is the cross-sectional 
area of that grain intersecting the vertical line, and $b$ is the width of the flume. The normalized, dimensionless sediment flux is then defined using the Einstein Number as $q^{*}=q_{s} /\left(D \sqrt{\left(\rho_{s}-\rho_{w}\right) g D / \rho_{w}}\right)$, where $D$ is grain diameter, $\rho_{s}$ is grain density, $\rho_{w}$ is the density of water, and $g$ is the acceleration due to gravity. This procedure was carried out for each video. The time-series from videos of the same experiment were then concatenated so as to have a single time series for each experiment. Although Figure 2 shows only two and a half seconds, most experiments have more than a minute of data, the lowest transport stages up to about 10 minutes.

For each experiment, we calculate the probability density function (PDF) of the time series $q^{*}(t)$. The PDFs all flattened for $q^{*}<10^{-3}$, which we considered to be the presence of measurement noise based on frame rate and video resolution. Therefore, all of our PDFs were truncated at around that value (see Figure 2g). The exponents of the PDF tails were calculated by performing a linear fit to the $\log \log$ plot of $\operatorname{PDF}\left(q^{*}\right)$ for small values of $q^{*}$, which we consider to be the tail. For the glass spheres, the interval in which the fit was performed was $10^{-3}<q^{*}<10^{-1}$, whereas for the natural grains it was $5 \times 10^{-3}<q^{*}<4 \times 10^{-1}$. The slope of this fit is the measured tail exponent, plotted in Figure 4. The error for the measured exponents were calculated by making a series of secondary fits over smaller intervals $d<q^{*}<d \times 10$, for $10^{-3}<d<10^{-2}$. The minimum slope found is the lower bound in the error bars and the maximum slope found is the upper bound.

Apart from taking the PDF of the sediment flux time series, a waiting time analysis was also performed. In this analysis an arbitrary fixed 'threshold' value of $q^{*}$ was chosen, $q_{0}^{*}$, and the waiting time $\Delta t$ between sediment transport events of size $q_{0}^{*}$ were measured. This is done in practice by increasing $\Delta t$ by one over the frame rate as long as $q^{*}<q_{0}^{*}$, and once $q_{0}^{*}$ is reached stopping the count, saving that $\Delta t$ and starting a count for a new $\Delta t$ in the sequence. Over a single time series, a sequence of waiting times $\left\{\Delta t_{i}\right\}$ were measured, and the PDF of this sequence was taken to get $P D F(\Delta t)$. For both experiments $q_{0}^{*}=0.05$. In theory, as long as $q_{0}^{*}$ is in the tail and the time series is long enough, any value of $q_{0}^{*}$ should give the same results ${ }^{42}$. However, values for $q_{0}^{*}$ lower than $10^{-3}$ run into issues with measurement noise, and a value larger than roughly $5 \times 10^{-1}$ result in a sequence of waiting times that is too small for good statistics, due to the limited length of the time series.

The curve fitting performed in both Figures 4 and 5 were done using the 'orthogonal distance regression' (ODR) method, part of the SciPy ${ }^{72}$ package for Python. This method accounts for errors in both variables, not just the 'dependent' variable like an ordinary least-squares regression. For each fit we specified the functional form being fit, with two free parameters to be estimated from the data. The ODR method results in both the values and standard errors of these estimated parameters. For Figure 4 we assumed a linear function, with the slope and intercept as the free parameters. For Figure 5 we assumed a function of the form of equation (4), with the free parameters being $\left\langle\tau^{*}\right\rangle_{c}$ and the constant of proportionality.

\section{Stochastic analysis}

Assuming that the sediment flux close to the threshold of motion is very small $\left(q^{*} \ll 1\right)$, we can approximate the nonlinear operator with a power series expansion in $\left\langle\tau^{*}\right\rangle-\left\langle\tau^{*}\right\rangle_{c}$ and $q^{*}$ as:

$$
\mathscr{N}\left(q^{*},\left\langle\tau^{*}\right\rangle-\left\langle\tau^{*}\right\rangle_{c}\right) \approx c_{00}+c_{10} q^{*}+c_{01}\left(\left\langle\tau^{*}\right\rangle-\left\langle\tau^{*}\right\rangle_{c}\right)+c_{11}\left(\left\langle\tau^{*}\right\rangle-\left\langle\tau^{*}\right\rangle_{c}\right) q^{*}+c_{20}\left(q^{*}\right)^{2}+c_{02}\left(\left\langle\tau^{*}\right\rangle-\left\langle\tau^{*}\right\rangle_{c}\right)^{2}+\cdots
$$

The coefficients of this expansion are then narrowed down using arguments about what kind of behavior is expected or observed. We assume that zero flux is a possible solution (stable or unstable) for all $\left\langle\tau^{*}\right\rangle-\left\langle\tau^{*}\right\rangle_{c}$, which means that $c_{00}=c_{01}=c_{02}=0$. Furthermore, we want to capture the threshold of motion, and so we want $q^{*}=0$ as a stable steady-state solution for $\left\langle\tau^{*}\right\rangle\left\langle\left\langle\tau^{*}\right\rangle_{c}\right.$ and some nonzero sediment flux as the stable steady-state solution for $\left.\left\langle\tau^{*}\right\rangle\right\rangle\left\langle\tau^{*}\right\rangle_{c}$. This is achieved by setting $c_{10}=0$ and letting $c_{20}<0$. Dividing all terms by $c_{11}$ yields a dimensionless time $t^{*} \equiv c_{11} t$ and a dimensionless pre-factor $\beta \equiv c_{20} / c_{11}$. Thus the resulting equation that can capture this behavior is $\mathscr{N}\left(q^{*}\right) \approx\left(\left\langle\tau^{*}\right\rangle-\left\langle\tau^{*}\right\rangle_{c}\right) q^{*}-\beta\left(q^{*}\right)^{2}$. To obtain the results in our study, it is not necessary to know $c_{11}$ and $\beta$. It is possible to estimate the value of $\beta$ in our experiments using the full PDF of $q^{*}$ (equation (6)), which is derived below. However, estimating $c_{11}$ is only possible via time-dependent statistics of $q^{*}$ and therefore it is not possible to estimate the value of $c_{11}$ given the steady-state results presented in this work.

An important final step in the development of our stochastic model is the inclusion of the noise, $\xi$, whose time-average is zero. The noise captures instantaneous deviations from the average bed conditions, since otherwise the effects of time-averaged quantities are included in the evolution equation of the instantaneous sediment flux. The noise could represent fluctuations of turbulent fluid motion or heterogeneities in the bed, all of which represent fluctuations in the local shear stress. The final model equation is the one seen in equation (1). In the absence of noise, equation (1) has two steady state solutions, $q^{*}=0$ and $q^{*}=\left\langle\tau^{*}\right\rangle-\left\langle\tau^{*}\right\rangle_{c}$, the former of which is stable for $\left\langle\tau^{*}\right\rangle\left\langle\left\langle\tau^{*}\right\rangle_{c} \text { but unstable for }\left\langle\tau^{*}\right\rangle\right\rangle^{*} \tau_{c}$. This can be shown by considering very small values of sediment flux, whereby we ignore the second term on the right hand side. Equation (1) then predicts exponential decay or growth at a rate $\left\langle\tau^{*}\right\rangle-\left\langle\tau^{*}\right\rangle_{c}$, depending on if the shear stress offset is negative or positive, respectively. In the latter case, the nonlinear term acts to stop the exponential growth and the other solution, which is stable, is approached.

Although it is nonlinear, equation (1) is still amenable to theoretical stochastic analysis, even when the fluctuations are large compared to mean quantities. Below we summarize the steps by which equation (1), a stochastic ordinary differential (ODE) 
equation which we interpret in the Stratonovich sense ${ }^{73}$, leads to the PDF of $q^{*}$ at steady state, equation (2). We also outline the reason for the shape of the waiting time PDF, equation (3).

We assume that the noise term in equation (1), $\xi$, is Gaussian white noise with zero mean and variance $2 S$. For this case there exists a standard procedure to arrive at the equation for the PDF of $q^{*}$, known as the Fokker-Planck equation ${ }^{73,74}$. The procedure can be thought of as taking a histogram of the many trajectories that result from the many possible realizations of the noise, if one were to imagine each realization of the noise as a different deterministic forcing that depends on time. The Fokker-Planck equation for the PDF of $q^{*}$ based on equation (1) can be shown to be ${ }^{74}$ :

$$
\frac{\partial P D F\left(q^{*}, t^{*}\right)}{\partial t^{*}}=-\frac{\partial}{\partial q^{*}}\left[\left(\left(\left\langle\tau^{*}\right\rangle-\left\langle\tau^{*}\right\rangle_{c}\right) q^{*}-\beta\left(q^{*}\right)^{2}+S q^{*}\right) P D F\left(q^{*}, t^{*}\right)\right]+S \frac{\partial^{2}}{\partial q^{* 2}}\left(q^{* 2} P D F\left(q^{*}, t^{*}\right)\right) .
$$

We look for steady state solutions of equation (5), $P D F\left(q^{*}, t^{*}\right)=P D F\left(q^{*}\right)$, where $\partial P D F / \partial t^{*}=0$. Solving equation (5) for $\operatorname{PDF}\left(q^{*}\right)$ based on the steadiness assumption gives the following distribution:

$$
\operatorname{PDF}\left(q^{*}\right)=\left(q^{*}\right)^{\left(\left\langle\tau^{*}\right\rangle-\left\langle\tau^{*}\right\rangle_{c}\right) / S-1} e^{-\beta q^{*} / S} N
$$

where $N$ is a normalization factor. One then arrives at equation (2) by noting that the exponential term is approximately equal to one for small $q^{*}$.

This analysis assumes that the noise $\xi$ is uncorrelated in time ('white noise') $46,47,74$. However, these results have more recently been expanded to the case when the noise has some non-zero correlation time $t_{c}^{*}$ ('colored noise') ${ }^{57,75}$, which is more physically relevant to sediment transport. In order for the analysis to be valid for colored noise, one must assume that $\sqrt{2 S} t_{c}^{*} \ll 1$, where $t_{c}^{*}$ is the correlation time of the noise ${ }^{57,73}$. The final PDF has a form that differs from (6), but the PDF tail for small $q^{*}$ is still given by equation (2). The full expression for $\operatorname{PDF}\left(q^{*}\right)$ for the colored noise, but for a cubic nonlinearity rather than a quadratic one, can be seen in Aumaitre et al. ${ }^{57}$.

The PDF of waiting times between transport events of a certain size (equation (3)) is the 'first return time' distribution of a biased random walk. This calculation for on-off intermittency was first performed by Heagy et al. ${ }^{42}$ for the case of white noise, and the colored noise case is discussed in the work of Aumaitre et al. ${ }^{57}$. The waiting time refers to how long the time series $q^{*}\left(t^{*}\right)$ spends below a value $q_{0}^{*}$ (the 'off' phase), and once $q^{*}>q_{0}^{*}$ (the 'on' phase) the counting stops. If the defined threshold $q_{0}^{*}$ is small enough that, during the off phase, the nonlinearity in equation (1) is negligible, the dynamics of the off phase are purely determined by the linearized version of equation (1), which can be expressed in terms of logarithms as

$$
\frac{d \log \left(q^{*}\right)}{d t^{*}} \approx\left\langle\tau^{*}\right\rangle-\left\langle\tau^{*}\right\rangle_{c}+\xi
$$

Therefore, for $\left\langle\tau^{*}\right\rangle=\left\langle\tau^{*}\right\rangle_{c}$ and $q^{*}<q_{0}^{*}$, the time series performs an unbiased random walk in logarithmic space. When the random walk approaches zero (corresponding to $q^{*} \sim 1$ ), the nonlinearity becomes important and acts as a barrier that prevents the random walk from reaching much larger values. Passing this barrier means that an on phase has begun. Therefore, the time interval that starts when the random walk falls below the nonlinear barrier and ends when the random walk exceeds the nonlinear barrier is the duration of an off phase. Thus, the distribution of waiting times is analogous to the well-established 'first return time' distribution of random walks, which specifies the distribution of times it takes for a random walk to return to a specified value. The first return time has a distribution $\Delta t^{-3 / 2}$. Due to the central limit theorem, this result is universal and holds for any kind of noise, as long as the mean is zero and the variance is finite. When $\left.\left\langle\tau^{*}\right\rangle\right\rangle^{*}\left\langle\tau^{*}\right\rangle_{c}$, the trajectory is no longer that of an unbiased random walker, and tends to favor going towards zero. This means that extremely long waiting times become less and less likely, and therefore alters the power law first return time distribution to have an exponential cut-off at large waiting times, resulting in equation (3).

It may seem counter-intuitive that the exponential cutoff of the distribution (equation (3)) depends only on $\left\langle\tau^{*}\right\rangle-\left\langle\tau^{*}\right\rangle_{c}$ and not on $q_{0}^{*}$. The reason for this is the assumption that $q_{0}^{*}$ is small enough that the nonlinearity in the dynamical equation, equation (1), is negligible when $q^{*} \sim q_{0}^{*}$. The return time therefore depends only on the dynamics in equation (7), which specifies that return time statistics depend not on the returning value, but rather on whether the walk is biased or not, which is determined by $\left\langle\tau^{*}\right\rangle-\left\langle\tau^{*}\right\rangle_{c}$.

\section{Data Availability}

The sediment flux time series data is available at https://doi.org/10.6084/m9.figshare.14450445.v1. For raw experimental data, contact the corresponding author. 


\section{Code Availability}

The python scripts used to analyze the raw experimental footage will be made available in a subsequent publication focusing broadly on the experimental data set, but for the time being anyone interested in the code can contact the corresponding author.

\section{Acknowledgements}

We thank Catherine Johnson and Mark Jellinek for logistical support. Research was sponsored by the Army Research Laboratory and was accomplished under Grant Number W911NF-16-1-0440. The views and conclusions contained in this document are those of the authors and should not be interpreted as representing the official policies, either expressed or implied, of the Army Research Laboratory or the U.S. Government. The U.S. Government is authorized to reproduce and distribute reprints for Government purposes notwithstanding any copyright notation herein. S. J. B also acknowledges funding from a grant from the National Science Foundation (OCE-1459702).

\section{Author contributions}

S. J. B. and E. A. D. conceived and developed the intermittent transport model. J. T. P., J. G. V., and K. K. conceived and designed the experiments. E. A. D., S. J. B., J. G. V., and J. T. P. conducted the experiments. E. A. D. and M. R. performed the particle tracking. E. A. D., S. J. B., and M. R. performed the data analysis. S. J. B. and J. T. P. wrote the manuscript with input from the other authors.

\section{Competing interests}

The authors declare no competing interests.

\section{References}

1. Bridge, J. \& Demicco, R. Earth Surface Processes, Landforms and Sediment Deposits (Cambridge University Press, 2008).

2. Anderson, R. S. \& Anderson, S. P. Geomorphology: the mechanics and chemistry of landscapes (Cambridge University Press, 2010).

3. Jones, D. K. C., Cooke, R. U. \& Warren, A. Geomorphological investigation, for engineering purposes, of blowing sand and dust hazard. Q. J. Eng. Geol. Hydrogeol. 19, 251-270, DOI: 10.1144/GSL.QJEG.1986.019.03.03 (1986). https://qjegh.lyellcollection.org/content/19/3/251.full.pdf.

4. Gilvear, D. J. Fluvial geomorphology and river engineering: future roles utilizing a fluvial hydrosystems framework. Geomorphology 31, 229 - 245, DOI: https://doi.org/10.1016/S0169-555X(99)00086-0 (1999).

5. Alcantara, I. \& Goudie, A. Geomorphological Hazards and Disaster Prevention. Cambridge books online (Cambridge University Press, 2010).

6. Wohl, E. et al. The natural sediment regime in rivers: Broadening the foundation for ecosystem management. BioScience 65, 358-371, DOI: 10.1093/biosci/biv002 (2015). https://academic.oup.com/bioscience/article-pdf/65/4/358/16648533/ biv002.pdf.

7. Wilcock, P. R. Stream Restoration in Gravel-Bed Rivers, chap. 12, 135-149 (John Wiley \& Sons, Ltd, 2012).

8. Simon, A., Bennett, S. J. \& Castro, J. M. Stream restoration in dynamic fluvial systems: Scientific approaches, analyses, and tools, vol. 194 (John Wiley \& Sons, 2013).

9. Meyer-Peter, E. \& Müller, R. Formulas for bed-load transport. In IAHSR 2nd meeting, Stockholm, appendix 2 (IAHR, 1948).

10. Einstein, H. A. The bed-load function for sediment transportation in open channel flows. No. 1026. US Dep. Agric. (1950).

11. Bagnold, R. A. The Flow of Cohesionless Grains in Fluids. Philos. Transactions Royal Soc. A: Math. Phys. Eng. Sci. 249 (1956).

12. Ashida, K. \& Michiue, M. Study on hydraulic resistance and bed-load transport rate in alluvial streams. In Proceedings of the Japan society of civil engineers, 206, 59-69 (Japan Society of Civil Engineers, 1972).

13. Engelund, F. \& Fredsøe, J. A sediment transport model for straight alluvial channels. Hydrol. Res. 7, 293-306, DOI: 10.2166/nh.1976.0019 (1976).

14. Luque, R. F. \& Beek, R. V. Erosion and transport of bed-load sediment. J. Hydraul. Res. 14, 127-144, DOI: 10.1080/ 00221687609499677 (1976). https://doi.org/10.1080/00221687609499677. 
15. Parker, G., Klingeman, P. \& McLean, D. Bedload and size distribution in paved gravel-bed streams. J. Hydraul. Eng. 108, 544-571 (1982).

16. Parker, G. Surface-based bedload transport relation for gravel rivers. J. Hydraul. Res. 28, 417-436, DOI: 10.1080/ 00221689009499058 (1990).

17. Wilcock, P. R. \& Crowe, J. C. Surface-based transport model for mixed-size sediment. J. Hydraul. Eng. 129, 120-128 (2003).

18. Wong, M. \& Parker, G. Reanalysis and correction of bed-load relation of Meyer-Peter and Müller using their own database. J. Hydraul. Eng. 132, 1159-1168, DOI: 10.1061/(ASCE)0733-9429(2006)132:11(1159) (2006).

19. Shields, A. Anwendung der aehnlichkeitsmechanik und der turbulenzforschung auf die geschiebebewegung. PhD Thesis Tech. Univ. Berlin (1936).

20. Parker, G. Self-formed straight rivers with equilibrium banks and mobile bed. part 2. the gravel river. J. Fluid Mech. 89, 127-146, DOI: 10.1017/S0022112078002505 (1978).

21. Parker, G., Wilcock, P. R., Paola, C., Dietrich, W. E. \& Pitlick, J. Physical basis for quasi-universal relations describing bankfull hydraulic geometry of single-thread gravel bed rivers. J. Geophys. Res. Earth Surf. 112, DOI: 10.1029/2006JF000549 (2007). https://agupubs.onlinelibrary.wiley.com/doi/pdf/10.1029/2006JF000549.

22. Buffington, J. M. \& Montgomery, D. R. A systematic analysis of eight decades of incipient motion studies, with special reference to gravel-bedded rivers. Water Resour. Res. 33, 1993-2029, DOI: 10.1029/96WR03190 (1997). https: //agupubs.onlinelibrary.wiley.com/doi/pdf/10.1029/96WR03190.

23. Stout, J. E. \& Zobeck, T. M. Intermittent saltation. Sedimentology 44, 959-970, DOI: 10.1046/j.1365-3091.1997.d01-55.x (1997).

24. Carneiro, M. V., Rasmussen, K. R. \& Herrmann, H. J. Bursts in discontinuous Aeolian saltation. Sci. Reports 5, DOI: 10.1038/srep11109 (2015). 1403.4840.

25. Houssais, M., Ortiz, C. P., Durian, D. J. \& Jerolmack, D. J. Onset of sediment transport is a continuous transition driven by fluid shear and granular creep. Nat. Commun. 6, 1-8, DOI: 10.1038/ncomms7527 (2015).

26. Gomez, B. Bedload transport. Earth-Science Rev. 31, 89-132, DOI: 10.1016/0012-8252(91)90017-A (1991).

27. Ancey, C., Böhm, T., Jodeau, M. \& Frey, P. Statistical description of sediment transport experiments. Phys. Rev. E 74, 1-14, DOI: 10.1103/PhysRevE.74.011302 (2006).

28. Ancey, C., Davison, A. C., Böhm, T., Jodeau, M. \& Frey, P. Entrainment and motion of coarse particles in a shallow water stream down a steep slope. J. Fluid Mech. 595, 83-114, DOI: 10.1017/S0022112007008774 (2008).

29. Singh, A., Fienberg, K., Jerolmack, D. J., Marr, J. \& Foufoula-Georgiou, E. Experimental evidence for statistical scaling and intermittency in sediment transport rates. J. Geophys. Res. Earth Surf. 114, 1-16, DOI: 10.1029/2007JF000963 (2009).

30. Heyman, J., Mettra, F., Ma, H. B. \& Ancey, C. Statistics of bedload transport over steep slopes: Separation of time scales and collective motion. Geophys. Res. Lett. 40, 128-133, DOI: 10.1029/2012GL054280 (2013).

31. Saletti, M., Molnar, P., Zimmermann, A., Hassan, M. A. \& Church, M. Temporal variability and memory in sediment transport in an experimental step-pool channel. Water Resour. Res. 51, 9325-9337, DOI: 10.1002/2015WR016929 (2015). https://agupubs.onlinelibrary.wiley.com/doi/pdf/10.1002/2015WR016929.

32. Pähtz, T. \& Durán, O. The Cessation Threshold of Nonsuspended Sediment Transport Across Aeolian and Fluvial Environments. J. Geophys. Res. Earth Surf. 123, 1638-1666, DOI: 10.1029/2017JF004580 (2018). 1602.07079.

33. Lee, D. B. \& Jerolmack, D. Determining the scales of collective entrainment in collision-driven bed load. Earth Surf. Dyn. 6, 1089-1099, DOI: 10.5194/esurf-6-1089-2018 (2018).

34. Liu, M. X., Pelosi, A. \& Guala, M. A Statistical Description of Particle Motion and Rest Regimes in Open-Channel Flows Under Low Bedload Transport. J. Geophys. Res. Earth Surf. 124, 2666-2688, DOI: 10.1029/2019JF005140 (2019).

35. Bunte, K. \& Abt, S. R. Effect of sampling time on measured gravel bed load transport rates in a coarse-bedded stream. Water Resour. Res. 41, DOI: 10.1029/2004WR003880 (2005). https://agupubs.onlinelibrary.wiley.com/doi/pdf/10.1029/ 2004WR003880.

36. Ancey, C., Bohorquez, P. \& Heyman, J. Stochastic interpretation of the advection-diffusion equation and its relevance to bed load transport. J. Geophys. Res. Earth Surf. 120, 2529-2551, DOI: 10.1002/2014JF003421 (2015). https: //agupubs.onlinelibrary.wiley.com/doi/pdf/10.1002/2014JF003421. 
37. Furbish, D. J., Haff, P. K., Roseberry, J. C. \& Schmeeckle, M. W. A probabilistic description of the bed load sediment flux: 1. Theory. J. Geophys. Res. Earth Surf. 117, DOI: 10.1029/2012JF002352 (2012).

38. Roseberry, J. C., Schmeeckle, M. W. \& Furbish, D. J. A probabilistic description of the bed load sediment flux: 2. particle activity and motions. J. Geophys. Res. Earth Surf. 117, DOI: 10.1029/2012JF002353 (2012). https://agupubs.onlinelibrary. wiley.com/doi/pdf/10.1029/2012JF002353.

39. Heyman, J., Ma, H. B., Mettra, F. \& Ancey, C. Spatial correlations in bed load transport: Evidence, importance, and modeling. J. Geophys. Res. Earth Surf. 119, 1751-1767, DOI: 10.1002/2013JF003003 (2014).

40. Ancey, C. \& Heyman, J. A microstructural approach to bed load transport: mean behaviour and fluctuations of particle transport rates. J. Fluid Mech. 744, 129-168, DOI: 10.1017/jfm.2014.74 (2014).

41. Platt, N., Spiegel, E. A. \& Tresser, C. On-off intermittency: A mechanism for bursting. Phys. Rev. Lett. 70, 279-282, DOI: 10.1103/PhysRevLett.70.279 (1993).

42. Heagy, J. F., Platt, N. \& Hammel, S. M. Characterization of on-off intermittency. Phys. Rev. E 49 (1994).

43. Ott, E. \& Sommerer, J. C. Blowout bifurcations: the occurrence of riddled basins and on-off intermittency. Phys. Lett. A 188, 39 - 47, DOI: https://doi.org/10.1016/0375-9601(94)90114-7 (1994).

44. Fujisaka, H. \& Yamada, T. A new intermittency in coupled dynamical systems. Prog. Theor. Phys. 74, 918 (1985).

45. Pikovsky, A. S. On the interaction of strange attractors. Zeitschrift für Physik B Condens. Matter 55, 149-154 (1984).

46. Horsthemke, W. \& Malek-Mansour, M. The influence of external noise on non-equilibrium phase transitions. Zeitschrift für Physik B Condens. Matter 24, 307-313 (1976).

47. Kabashima, S., Kogure, S., Kawakubo, T. \& Okada, T. Oscillatory-to-nonoscillatory transition due to external noise in a parametric oscillator. J. Appl. Phys. 50, 6296-6302, DOI: 10.1063/1.325769 (1979). https://doi.org/10.1063/1.325769.

48. Hammer, P. W., Platt, N., Hammel, S. M., Heagy, J. F. \& Lee, B. D. Experimental observation of on-off intermittency. Phys. Rev. Lett. 73, 1095-1098 (1994).

49. Feng, D. L., Yu, C. X., Xie, J. L. \& Ding, W. X. On-off intermittencies in gas discharge plasma. Phys. Rev. E 58, 3678 (1998).

50. John, T., Stannarius, R. \& Behn, U. On-off intermittency in stochastically driven electrohydrodynamic convection in nematics. Phys. Rev. Lett. 83, 749 (1999).

51. Sweet, D., Ott, E., Finn, J. M., Antonsen, T. M. \& Lathrop, D. P. Blowout bifurcations and the onset of magnetic activity in turbulent dynamos. Phys. Rev. E 63, 066211, DOI: 10.1103/PhysRevE.63.066211 (2001).

52. Bottiglieri, M. \& Godano, C. On-off intermittency in earthquake occurrence. Phys. Rev. E 75, 026101, DOI: 10.1103/ PhysRevE.75.026101 (2007).

53. Alexakis, A. \& Ponty, Y. Effect of the lorentz force on on-off dynamo intermittency. Phys. Rev. E 77, 056308, DOI: 10.1103/PhysRevE.77.056308 (2008).

54. Benavides, S. J. \& Alexakis, A. Critical transitions in thin layer turbulence. J. Fluid Mech. 822, 364-385, DOI: 10.1017/jfm.2017.293 (2017).

55. Crawford, J. D. Introduction to bifurcation theory. Rev. Mod. Phys. 63, 991-1037, DOI: 10.1103/RevModPhys.63.991 (1991).

56. Kardar, M. Statistical physics of fields (Cambridge University Press, 2007).

57. Aumaître, S., Mallick, K. \& Pétrélis, F. Effects of the low frequencies of noise on on-off intermittency. J. Stat. Phys. 123, 909-927, DOI: 10.1007/s10955-006-9116-9 (2006). 0707.2456.

58. Bradley, R. W. \& Venditti, J. G. The growth of dunes in rivers. J. Geophys. Res. Earth Surf. 124, 548-566, DOI: https://doi.org/10.1029/2018JF004835 (2019). https://agupubs.onlinelibrary.wiley.com/doi/pdf/10.1029/2018JF004835.

59. Venditti, J. G., Nelson, P. A., Bradley, R. W., Haught, D. \& Gitto, A. B. Bedforms, structures, patches, and sediment supply in gravel-bed rivers. Gravel-Bed Rivers: Process. Disasters; Tsutsumi, D., Laronne, JB, Eds 439-466 (2017).

60. Miller, M. C., McCave, I. N. \& Komar, P. D. Threshold of sediment motion under unidirectional currents. Sedimentology 24, 507-527, DOI: 10.1111/j.1365-3091.1977.tb00136.x (1977). https://onlinelibrary.wiley.com/doi/pdf/10.1111/j.1365-3091. 1977.tb00136.x.

61. Yalin, M. S. \& Karahan, E. Inception of sediment transport. J. hydraulics division 105, 1433-1443 (1979). 
62. Pétrélis, F. \& Alexakis, A. Anomalous exponents at the onset of an instability. Phys. Rev. Lett. 108, 1-5, DOI: 10.1103/PhysRevLett.108.014501 (2012). 1112.0999v1.

63. Alexakis, A. \& Pétrélis, F. Critical exponents in zero dimensions. J. Stat. Phys. 149, 738-753, DOI: 10.1007/ s10955-012-0615-6 (2012). 1207.4355v1.

64. Young, W. \& Warburton, J. Principles and practice of hydraulic modelling of braided gravel-bed rivers. J. Hydrol. (New Zealand) 35, 175-198 (1996).

65. Dudill, A., Venditti, J. G., Church, M. \& Frey, P. Comparing the behaviour of spherical beads and natural grains in bedload mixtures. Earth Surf. Process. Landforms 45, 831-840, DOI: 10.1002/esp.4772 (2020). https://onlinelibrary.wiley.com/ doi/pdf/10.1002/esp.4772.

66. Bradski, G. The OpenCV Library. Dr. Dobb's J. Softw. Tools (2000).

67. Bradski, G. \& Kaehler, A. Learning OpenCV: Computer vision with the OpenCV library (" O’Reilly Media, Inc.", 2008).

68. Afik, E. Robust and highly performant ring detection algorithm for $3 \mathrm{~d}$ particle tracking using $2 \mathrm{~d}$ microscope imaging. Sci. Reports 5, 13584, DOI: 10.1038/srep13584 (2015).

69. Allan, D. et al. soft-matter/trackpy: Trackpy v0.4.2, DOI: 10.5281/zenodo.3492186 (2019).

70. soft-matter/pims: Pims v0.5. https://github.com/soft-matter/pims (2020).

71. Redmon, J., Divvala, S., Girshick, R. \& Farhadi, A. You only look once: Unified, real-time object detection. In Proceedings of the IEEE conference on computer vision and pattern recognition, 779-788 (2016).

72. Virtanen, P. et al. SciPy 1.0: Fundamental Algorithms for Scientific Computing in Python. Nat. Methods 17, 261-272, DOI: $10.1038 / \mathrm{s} 41592-019-0686-2$ (2020).

73. Van Kampen, N. G. Stochastic processes in physics and chemistry, vol. 1 (Elsevier, 1992).

74. Schenzle, A. \& Brand, H. Multiplicative stochastic processes in statistical physics. Phys. Rev. A 20, 1628-1647, DOI: 10.1103/PhysRevA.20.1628 (1979).

75. Aumaître, S., Pétrélis, F. \& Mallick, K. Low-frequency noise controls on-off intermittency of bifurcating systems. Phys. Rev. Lett. 95, 2-5, DOI: 10.1103/PhysRevLett.95.064101 (2005). 0608197. 


\section{Supplementary Data and Figures}
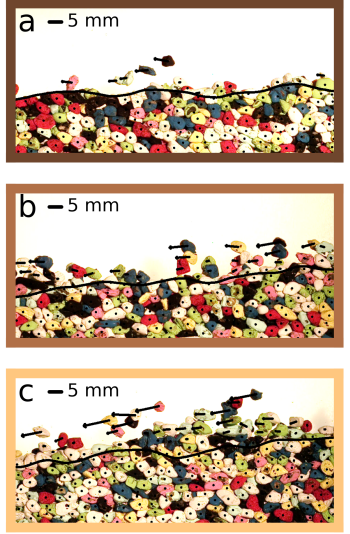
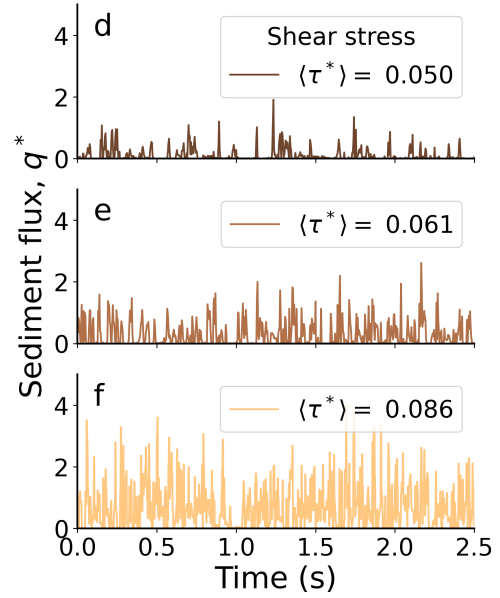

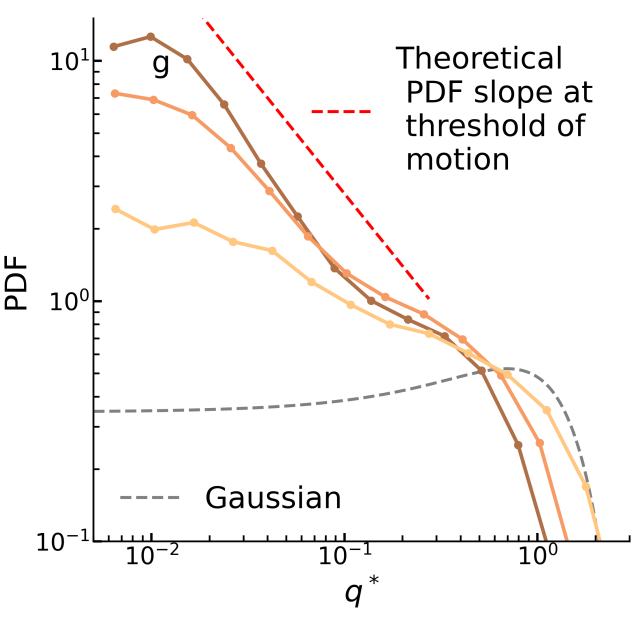

Extended Data Fig. 1. Intermittent bed load sediment flux. Sample data from three flume experiments using natural grains, representing typical runs with low sediment flux (a and $\mathbf{d}$ ), intermediate sediment flux (b and $\mathbf{e}$ ), and high sediment flux $(\mathbf{c}$ and $\mathbf{f})$. a-c, High-speed video frames showing grain centers and velocities and the location of the bed, as in Figure 1c. d-f, Samples of the corresponding sediment flux time series. g, Probability density function (PDF) calculated from the sediment flux time-series. The red dashed line shows the theoretical slope of the PDF tail at the threshold of motion. The grey dashed line shows a Gaussian distribution with the same mean and variance as the high sediment flux case. 

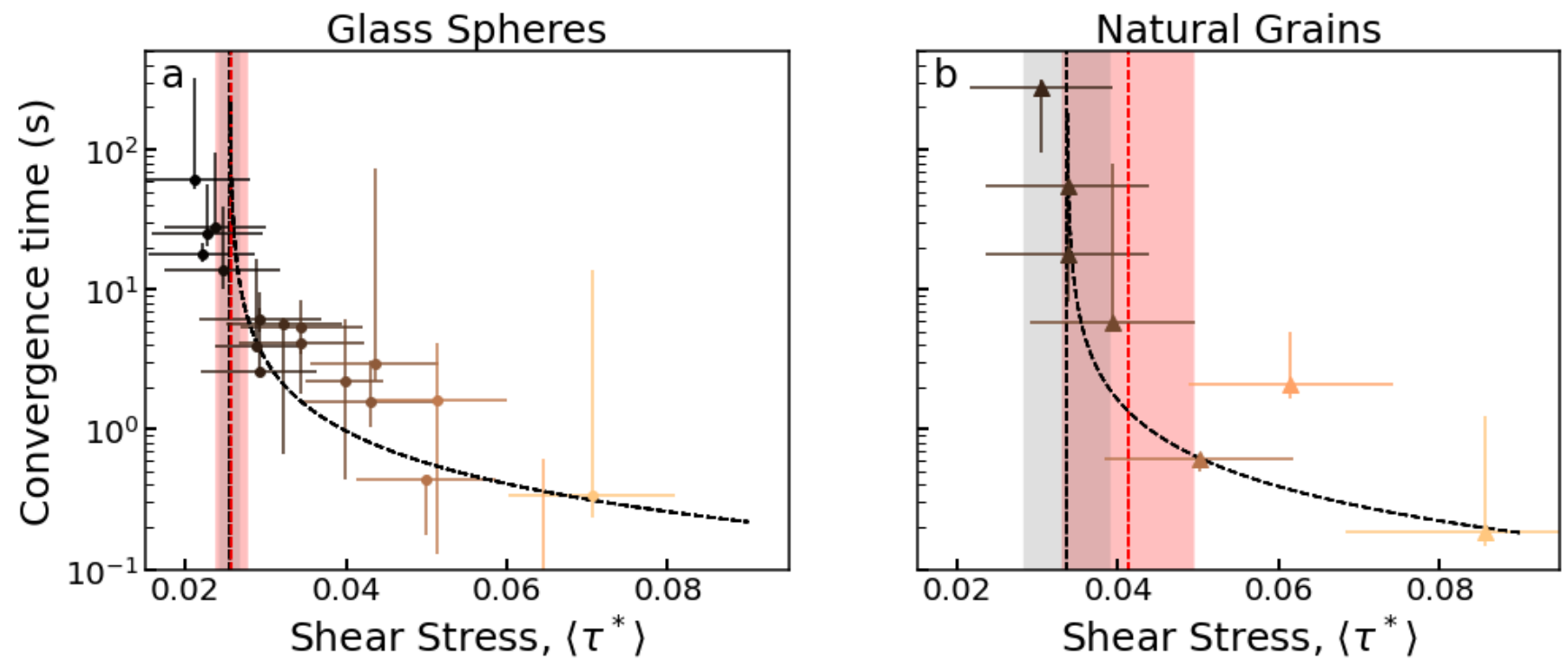

Extended Data Fig. 2. Convergence time for the average sediment flux. The averaging time necessary to converge to within $15 \%$ of the final average for various values of shear stress $\left\langle\tau^{*}\right\rangle$ in the experiments with $\mathbf{a}$ glass spheres, and $\mathbf{b}$ natural grains. Point colors correspond to the shear stress color scale in Figure 3. Uncertainty bars in the shear stress are one standard error of the mean. Uncertainty bars in the convergence time represent the time needed to converge to within $20 \%$ of the final average (lower limit) and within 10\% of the final average (upper limit). Dashed black curves are fits of equation (4) to the data using an orthogonal distance regression. Values of the critical shear stress, $\left\langle\tau^{*}\right\rangle_{c}$, determined from the fits are denoted by vertical black dashed lines, with grey shading indicating one standard error. Red dashed lines with rose shading indicate the critical shear stress determined from the PDF of $q^{*}$ (Figure 4). 

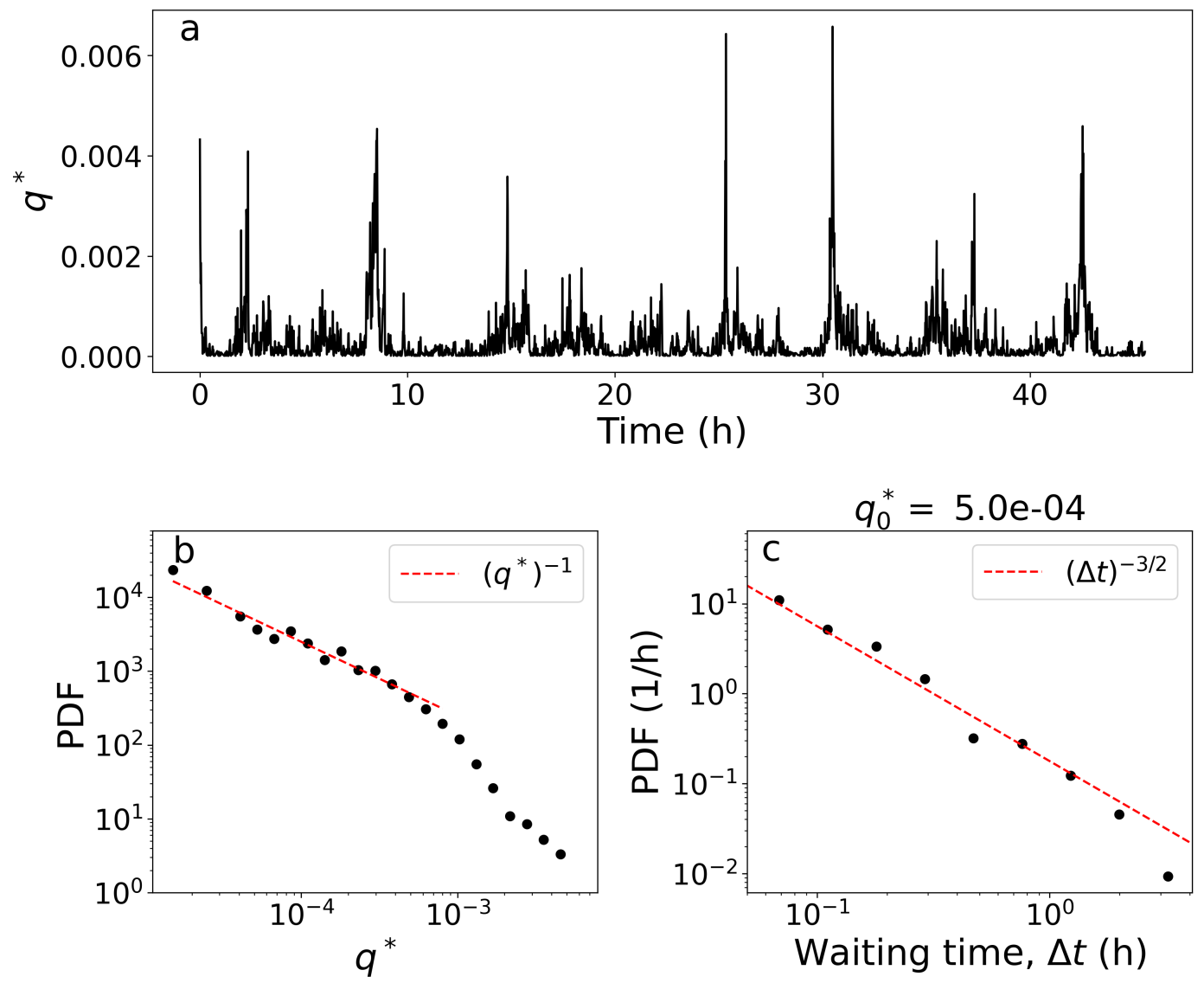

Extended Data Fig. 3. Data from the experiments of Ancey et al. ${ }^{36}$ Intermittent sediment flux time series taken from an experiment performed by Ancey et al. ${ }^{36}$ in a flume $2.5 \mathrm{~m}$ long and $8 \mathrm{~cm}$ wide, using gravel with mean diameter $d_{50}=6.25 \mathrm{~mm}$, feed rate of approximately $q_{i n}^{*}=0.0003$, or 0.37 particles per second, and average water depth of $h=1.37 \mathrm{~cm}$ so that $h / d_{50}=2.2$. This experiment was performed for over 50 hours and produced intermittent bursts of transport orders of magnitude larger than the input rate (a). Both the PDF of $q^{*}(\mathbf{b})$ and of the waiting time (c) are consistent with our findings, which state that the tail exponent for the PDF of $q^{*}$ should be approximately -1 (equation (2)) and that the waiting time distribution PDF should be a power law with exponent $-3 / 2$ (equation (3)). Despite the average waiting time of only $\langle\Delta t\rangle=6$ mins between transport events of $q_{0}^{*}=5 \times 10^{-4}$, the convergence time for a correctly determined average was found to be 10 hours ${ }^{36}$. This factor of 100 ratio between average waiting time and convergence time is similar to our experimental findings. 

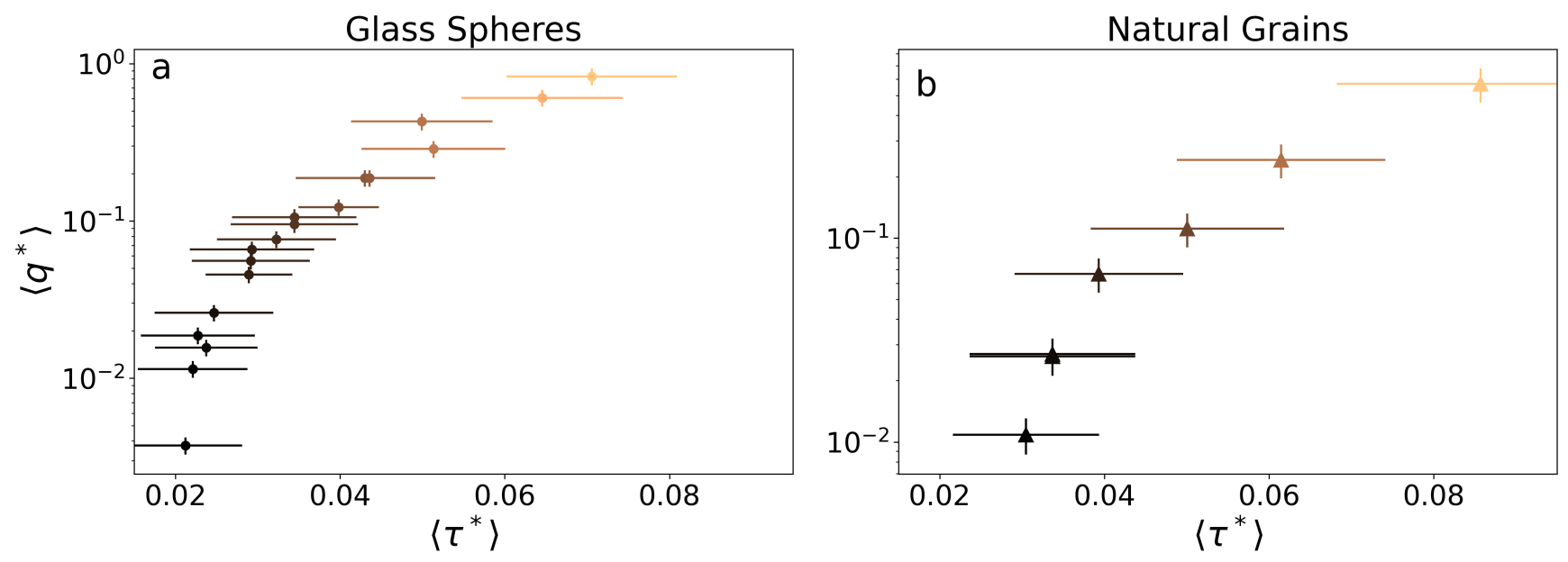

Extended Data Fig. 4. Average sediment flux. Time-averaged sediment flux for every experiment performed for this study using (a) glass spheres and (b) natural grains. 